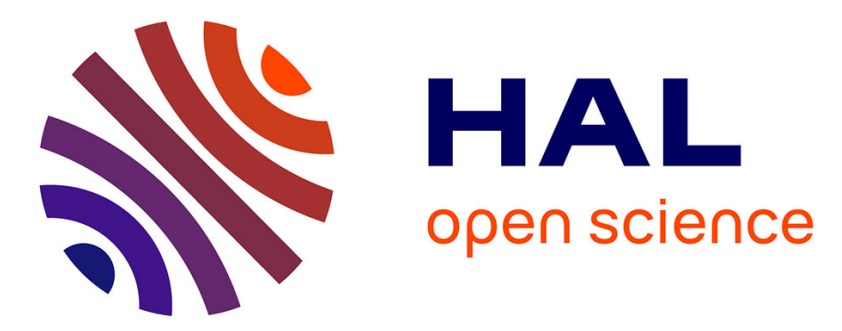

\title{
Automaticity of phonological and semantic processing during visual word recognition
}

Chotiga C Pattamadilok, Valérie C Chanoine, Christophe C Pallier, Jean-Luc C Anton, Bruno C Nazarian, Pascal C Belin, Johannes C. Ziegler

\section{To cite this version:}

Chotiga C Pattamadilok, Valérie C Chanoine, Christophe C Pallier, Jean-Luc C Anton, Bruno C Nazarian, et al.. Automaticity of phonological and semantic processing during visual word recognition. NeuroImage, 2017, 149, pp.244 - 255. 10.1016/j.neuroimage.2017.02.003 . hal-01481043

\section{HAL Id: hal-01481043 \\ https://hal-amu.archives-ouvertes.fr/hal-01481043}

Submitted on 2 Mar 2017

HAL is a multi-disciplinary open access archive for the deposit and dissemination of scientific research documents, whether they are published or not. The documents may come from teaching and research institutions in France or abroad, or from public or private research centers.
L'archive ouverte pluridisciplinaire HAL, est destinée au dépôt et à la diffusion de documents scientifiques de niveau recherche, publiés ou non, émanant des établissements d'enseignement et de recherche français ou étrangers, des laboratoires publics ou privés. 


\title{
Automaticity of phonological and semantic processing during visual word recognition
}

\author{
Chotiga Pattamadilok ${ }^{\mathrm{a}, *}$, Valérie Chanoine ${ }^{\mathrm{b}}$, Christophe Pallier ${ }^{\mathrm{c}}$, Jean-Luc Anton ${ }^{\mathrm{d}}$, \\ Bruno Nazarian ${ }^{\mathrm{d}}$, Pascal Belin ${ }^{\mathrm{d}}$, Johannes C. Ziegler ${ }^{\mathrm{e}}$ \\ a Aix Marseille Univ, CNRS, LPL, Aix-en-Provence, France \\ b Labex Brain and Language Research Institute, Aix-en-Provence, France \\ ' INSERM-CEA Cognitive Neuroimaging Unit, Neurospin center, Gif-sur-Yvette, France \\ a Aix Marseille Univ, CNRS, INT Inst Neurosci Timone, UMR 7289, Centre IRM Fonctionnelle Cérébrale, Marseille, France \\ e Aix Marseille Univ, CNRS, LPC, Marseille, France
}

\section{A R T I C L E I N F O}

\section{Keywords:}

Automatic activation

Bottom-up process

Stimulus-driven

Task-dependent

Top-down process

\begin{abstract}
A B S T R A C T
Reading involves activation of phonological and semantic knowledge. Yet, the automaticity of the activation of these representations remains subject to debate. The present study addressed this issue by examining how different brain areas involved in language processing responded to a manipulation of bottom-up (level of visibility) and top-down information (task demands) applied to written words. The analyses showed that the same brain areas were activated in response to written words whether the task was symbol detection, rime detection, or semantic judgment. This network included posterior, temporal and prefrontal regions, which clearly suggests the involvement of orthographic, semantic and phonological/articulatory processing in all tasks. However, we also found interactions between task and stimulus visibility, which reflected the fact that the strength of the neural responses to written words in several high-level language areas varied across tasks. Together, our findings suggest that the involvement of phonological and semantic processing in reading is supported by two complementary mechanisms. First, an automatic mechanism that results from a taskindependent spread of activation throughout a network in which orthography is linked to phonology and semantics. Second, a mechanism that further fine-tunes the sensitivity of high-level language areas to the sensory input in a task-dependent manner.
\end{abstract}

\section{Introduction}

Reading is a multimodal activity. Many studies indeed show that processing written words engages not only orthographic but also phonological and semantic processes (Kiefer and Martens, 2010; Mechelli et al., 2007; Van Orden, 1987; Wheat et al., 2010; Wilson et al., 2011). In the context of interactive connectionist models of word perception, this observation is explained by a spreading of activation throughout a network in which orthography is linked to phonological and semantic information via weighted connections. Such spreading of activation provides the core mechanism for reading models that postulate an automatic activation of phonological and semantic representations in response to written input (Harm and Seidenberg, 2004, 1999; Seidenberg and McClelland, 1989; Van Orden and Goldinger, 1994).

Yet, empirical evidence supporting the automaticity assumption remains controversial. On the one hand, several studies reported that phonological and semantic activation can be blocked or modulated by attentional and task demands, thus suggesting some form of top-down influence of the high-level processes involved in word perception (Brown et al., 2001; Demonet et al., 1992; Devlin et al., 2003; McDermott et al., 2003; Poldrack et al., 1999; Rumsey et al., 1997). On the other hand, this attentional and task-dependent account has been questioned by findings from a number of psycholinguistic studies that reported phonological and semantic effects in visual word recognition even when these representations were totally irrelevant to the task or not directly accessible (Rodd, 2004; Tanenhaus et al., 1980; Ziegler and Jacobs, 1995), thus supporting the claim of an automatic and possibly mandatory access to phonology and meaning during reading (Frost, 1998). Similarly, masked priming studies showed that shared phonological and semantic representations between a prime and a target affect recognition of the target even in the absence of prime awareness, which makes the strategic activation of these representations unlikely (Brysbaert, 2001; Brysbaert et al., 1999; Deacon et al.,

\footnotetext{
* Correspondence to: Aix Marseille Univ, CNRS, Laboratoire Parole et Langage, UMR 7309, 5, Av. Pasteur, Aix-en-Provence 13100, France.

E-mail address: chotiga.pattamadilok@lpl-aix.fr (C. Pattamadilok).
} 
2000; Drieghe and Brysbaert, 2002; Ferrand and Grainger, 1994; Kiefer and Spitzer, 2000; Lukatela and Turvey, 1994; Ortells et al., 2016; Wheat et al., 2010).

Thus, the question remains as to whether phonological and semantic activation in written word processing is task-dependent or whether it occurs automatically whenever participants process a written word. Although this topic has been extensively studied at the behavioral level (Besner et al., 1997; Labuschagne and Besner, 2015), the contribution of brain imaging studies to the debate is relatively scarce. The present study investigated how different brain areas involved in the processing of orthographic, phonological and semantic information responded to a manipulation of bottom-up and top-down information applied to written words.

So far, only a few brain imaging studies have manipulated bottomup and top-down factors within the same experiment. Among these studies, bottom-up factors have been mainly manipulated by comparing the activation patterns induced by different types of visual input ranging from checkerboards, objects, symbols, sequences of characters, pseudowords to real words (Carreiras et al., 2007; Twomey et al., 2011; Yang et al., 2012). As described below, the present study adopted a different approach that consisted in manipulating the bottom-up information while using only written words, i.e., visual stimuli that can potentially lead to the activation of orthographic, phonological and semantic information. Additionally, previous studies that used words mainly focused on the neural responses within specific brain areas (mainly within the visuo-orthographic system). This does not allow one to have a more global picture about what areas respond to orthographic, phonological and semantic information in a bottom-up versus top-down fashion.

The present study relied on the assumption that automatic responses would be driven by stimulus, or bottom-up information, independently of top-down information determined by task demand. Precisely, we used a go/no-go paradigm, in which participants either focused on visual (symbol detection), phonological (rime detection) or semantic content (animal name detection) of written words. The presence of the task effect would imply that a given brain area is activated in a task-dependent manner. In contrast to most previous fMRI studies, only words were used as visual input. Stimulus-driven (bottom-up) processes were manipulated by changing stimulus visibility parametrically. That is, the time between the stimulus and the visual masks was gradually increased such that the stimulus became progressively visible (Fig. 1) (Dehaene et al., 2001). This allowed us to manipulate the degree of visibility of the words while keeping their

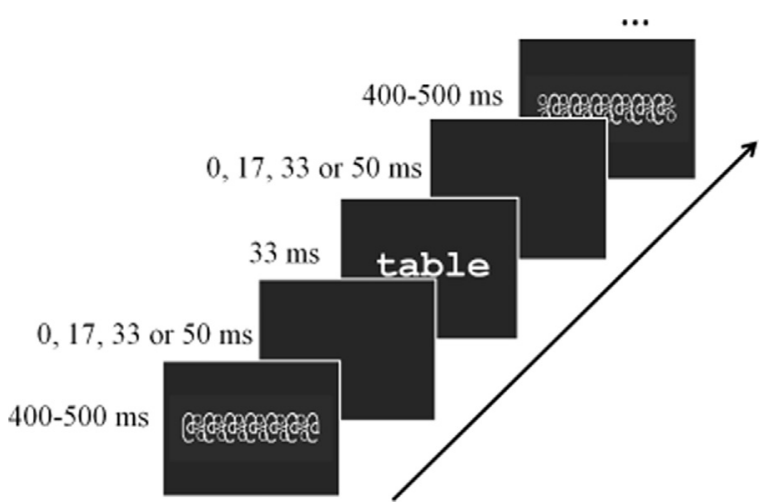

Fig. 1. An illustration of the timed-sequence of a word presented between forward and backward masks. Each trial consisted of four words presented at the same level of visibility as defined by the temporal distance between the words and the masks $(0,17,33$ or $50 \mathrm{~ms}$ for the L0, L1, L2 and L3 level of visibility, respectively). The duration of the words was kept constant (33 ms). presentation duration constant (Kouider et al., 2007). Participants' sensitivity to this manipulation was measured through a behavioral forced-choice task performed after the fMRI scan.

Given that reading also strongly relies on the activation of brain areas involved in spoken language (Rueckl et al., 2015), analyses were extended to several brain areas involved in spoken language processing. To tap the spoken language network independent of written input, we used auditory functional localizers to identify, at the subject-specific level, the brain areas that process the phonological and semantic aspects of spoken input and further tested whether the very same areas also responded to written words (Fedorenko et al., 2010).

Overall, we hypothesized that different brain areas might respond to bottom-up and top-down information in different ways. While highlevel language areas were expected to be more sensitive to task demands, the neural response of lower-level primary areas should be more sensitive to the visibility of the input. Interactions between task and visibility were expected in most areas of the reading network, although the precise pattern was thought to vary with the functional role of each specific area.

\section{Materials and methods}

\section{Participants}

Twenty-five young adults ( 14 women; age: mean $=23$ years; range $=$ 18-30 years) participated in the experiment. All were right-handed native speakers of French. The experiment was approved by the ethics committee for biomedical research, and written informed consent was obtained from all participants. They received $60 €$ for their participation. Two subjects were excluded from the analyses due to excessive head movements and a technical problem during the acquisition.

\section{Stimuli}

The critical stimuli, used in Nogo trials, were constructed in the same way in the symbol, rime and animal name detection tasks. Each trial consisted of an identical timed-sequence of four mono- or disyllabic French words, containing 4-7 letters, presented between forward and backward masks. Thus, within a trial, the four words were presented sequentially at the same level of visibility, defined by the temporal distance between the words and the masks as illustrated in Fig. 1. In the L0, least visible, condition, the forward and the backward masks immediately preceded and followed the words. In the L1, L2 and L3 condition, masks were separated from the words by $17 \mathrm{~ms}, 33 \mathrm{~ms}$ and $50 \mathrm{~ms}$, respectively. At all levels of visibility, word duration was kept constant at $33 \mathrm{~ms}$. Mask duration varied between 400 and $500 \mathrm{~ms}$ such that the duration of the trials always corresponded to $2.64 \mathrm{~s}$. Within participants, each word was presented at the four levels of visibility but only in one task. Across participants, each word was presented in all tasks. Stimuli were always presented at the center of the screen in Courier font. The largest visual angle was $12.1^{\circ}$.

Go trials were constructed in the same way as Nogo trials, excepted that one of the four words corresponded to the Go criterion, i.e., it contained the symbol "\&" (e.g., ma\& son), ended with the rime /o/ (e.g., château), or represented an animal name (e.g., lion) in the symbol, rime and animal name detection task, respectively. In each task, the Go trials were presented at the L2 or L3 level of visibility.

In addition to the Go and Nogo trials, we also included in each task "mask-only" trials in which the four words were replaced by blank screens. Although, no words were presented during the mask-only trials, the same four timed-sequences as the Nogo trials were used. The mask-only trials provide a low-level baseline condition (Dehaene et al., 2001; Kouider et al., 2007). 
Together, each task contained 32 Nogo trials presented at each of the four levels of visibility, 6 Go trials presented at L2 and L3 levels of visibility and 32 mask-only trials. Each type of trial was equally divided into two blocks that were presented in two runs.

\section{Procedure}

\section{Three main tasks}

Participants were imaged in six runs, corresponding to two runs per task. Task order was counter-balanced across subjects with a constraint that each task was presented only once within the first three runs. Within each run, the Go, Nogo and mask-only trials of different levels of visibility were presented in a pseudo-random order, respecting a calculation of efficiency. The ISI was jittered following an exponential curve to maximize design efficiency (ISI mean $=2 \mathrm{~s}$; ISI $\min =1 \mathrm{~s}$, see Henson (2015) for further description of design efficiency). Each run started with a short instruction indicating which task the participant had to perform next ("symbol", "rime" or "animal name" detection). The participants were required to push the response button with their right index as soon as they detected a Go trial. Before the experiment, short familiarization blocks were presented to the participants outside the scanner. The six runs lasted about $40 \mathrm{~min}$ in total.

\section{Localizer}

To identify the brain areas involved in acoustic, phonological and semantic processing of spoken sentences, participants were presented with short blocks of meaningful sentences, pseudo-sentences (sentences constructed from pseudowords) and scrambled sentences (sounds created by randomly shuffling phase and amplitude components in Fourier space of the pseudo-sentences in 21-ms windows, resulting in similar amplitude waveforms as the pseudo-sentences but a disrupted spectrum, unrecognizable as speech; cf., Belin et al., 2002). A block of trials contained seven sentences of the same category and lasted $12 \mathrm{~s}$ to $13 \mathrm{~s}$. It was followed either by a short silent period of $1.5 \mathrm{~s}$ to $2 \mathrm{~s}$ or by a long silent period of $13.4 \mathrm{~s}$. Together, there were six blocks for each type of sentence and no sentence was repeated. The 18 blocks were presented in a pseudo-random order to maximize design efficiency (Henson, 2015). During image acquisition, participants were simply required to listen to the sentences. The localizer lasted $6 \mathrm{~min}$.

\section{Behavioral forced-choice task}

At the end of the imaging session, while the participants were still lying in the scanner, they were tested behaviorally in a forced-choice task to individually evaluate their sensitivity to the stimuli presented at the different levels of visibility. Within each trial, participants saw a word presented between a forward and a backward mask. The distance between the word and the masks was manipulated as in the main tasks (Fig. 1). On each trial, the total duration of the word and the masks was $1.04 \mathrm{~s}$. After the presentation of the masked word, two words were presented simultaneously on the left and right of a fixation cross. One of them corresponded to the masked word and could appear on the left or right side with the same probability. Participants had to decide which word corresponded to the masked word by pushing one of the response buttons placed under their right index (for the left-side choice) and right major (for the right-side choice). As recommended in Kouider et al. (2007), the indexes of partial versus full awareness of the masked words could be obtained by manipulating the orthographic similarity of the two choices. Thus, on half of the trials, the two choices were orthographically distinct ('no overlap' trials, e.g., 'lune' vs. 'froc') while, on the other half, they shared at least $50 \%$ of the letters ('partial overlap' trials, e.g., 'aile' vs. 'aire'). Participants were previously trained outside the scanner with a short block of stimulus. The task contained 40 trials for each level of visibility (20 trials with partial overlap and 20 trials with no overlap). The trials were presented in a random order. The task lasted about $10 \mathrm{~min}$.

\section{Data acquisition and preprocessing}

Data acquisition was performed on a 3-T MEDSPEC 30/80 AVANCE imager (Bruker, Eittlingen, Germany) at the fMRI centre of the Institute of Neuroscience of La Timone, Marseille, France. A fieldmap acquisition (3D FLASH sequence inter-echo time $4.552 \mathrm{~ms}$ ) was collected in order to estimate and correct the B0 inhomogeneity. Functional images were acquired using a $\mathrm{T} 2{ }^{*}$-weighted gradient-echo planar sequence with 36 interleaved $3 \mathrm{~mm}$-thick/1 mm-gap slices (repetition time $=2.4 \mathrm{~s}$, echo time $=30 \mathrm{~ms}$, field of view $=192 \mathrm{~mm}, 64 \times 64$ matrix of $3 \times 3 \times 3 \mathrm{~mm}$ voxels). During the main tasks, a total of 1080 functional scans were acquired during six runs of 180 scans each. During the localizers, 140 functional scans were acquired. Whole brain anatomical MRI data was acquired during the behavioural forcedchoice task using high-resolution structural T1-weighted image (MPRAGE sequence, resolution $1 \times 1 \times 1 \mathrm{~mm}$ ) in the sagittal plane.

The fMRI data were pre-processed and analyzed using SPM8 software (Welcome Institute of Cognitive Neurology, London, UK). The first four volumes of each run were discarded to ensure that the longitudinal relaxation time equilibration was achieved. The anatomical scan was spatially normalized to the avg152 T1-weighted brain template defined by the Montreal Neurological Institute using the default parameters (nonlinear transformation). Functional volumes were corrected for slice timing differences, realigned, spatially normalized (using the combination of deformation field from T1 template, coregistered structural and sliced functional images) and smoothed with an isotropic Gaussian kernel $(\mathrm{FWHM}=6 \mathrm{~mm})$. The fieldmap images were used during the realign and unwarp procedure for distortion and motion correction.

\section{Brain imaging analyses}

\section{Whole brain analyses}

For each subject, a general linear model was generated. It included, for each of the two runs per task, 18 regressors modelling the 12 combinations of task and level of visibility on the Nogo trials (i.e., visual/L0; visual/L1; visual/L2; visual/L3; phone/L0; phone/L1; phone/L2; phone/L3; sem/L0; sem/L1; sem/L2; sem/L3), the three mask-only (baseline) conditions (one per task) and the three types of Go trials with motor response required (one per task). The eventrelated regressors used the canonical hemodynamic responses included in SPM8. Data were high-pass filtered with a cut-off of $128 \mathrm{~s}$. Six motion regressors corresponding to translation and rotation in each xyz were included in the design matrix.

For the group analysis, individual contrast maps representing coefficients to the 12 regressors (three tasks by four levels of visibility) of the Nogo trials and the three regressors of the mask-only trials were smoothed with a Gaussian filter (FWHM of $8 \mathrm{~mm}$ ). ${ }^{1}$ They were entered in an analysis of variance model (one-way ANOVA within subject design in SPM8) with one regressor per subject.

\section{Subject-specific ROI analyses}

In the last part of the Result section, we examined the brain activation within the spoken language system by performing analyses in regions of interest (ROIs), using the subject-specific approach proposed by Nieto-Castañón and Fedorenko (2012). The individual ROIs were obtained from independent localizers allowing us to identify the areas involved in acoustic, phonological and semantic processing of spoken sentences. These three types of functional ROIs were identified by contrasting scrambled sentences with silence baseline, pseudo-

\footnotetext{
${ }^{1}$ Smoothing the functional images stabilizes variance and improves tSNR and detection at the single subject level. Smoothing the individual contrast maps before entering them in the group analysis aims at increasing the spatial overlap between subjects. Smoothing in two stages at 6 and $8 \mathrm{~mm}$ results in an effective smoothing of $10 \mathrm{~mm}\left(\operatorname{sqrt}\left(6^{*} 6+8^{*} 8\right)\right)$ for the group analysis.
} 
Table 1

Description of the performance (in percentages) obtained in the three main tasks.

\begin{tabular}{lll}
\hline & Hit & False Alarm \\
\hline Visual task & 49 & 5 \\
Phonological task & 64 & 6 \\
Semantic task & 59 & 8 \\
\hline
\end{tabular}

sentences with scrambled sentences and meaningful sentences with pseudo-sentences, respectively. For each subject and each localizer contrast, only the voxels activated at the statistical threshold of $\mathrm{p}<.001$ voxelwise, uncorrected were included in the ROI definition. Data extraction and statistical analyses on the contrasts of interest were performed with the subject-specific (spm_ss) toolbox developed by Fedorenko et al. (2010).

\section{Results}

\section{Behavioral data}

As mentioned in the Introduction, the Go trials had been included to engage the participants in visual, phonological or semantic processes. Given that there were only 12 Go trials within each task, no statistical analysis was run on the data. Table 1 provided a description of the performance obtained in each task. The global performance pattern was similar across the three tasks. Hit rates were generally low, with the lowest rate observed in the visual task. This might be due to the difficulty to distinguish the symbol " \&" from real letters when fast presentation rates were used. However, low false alarm rates (5-8\%) argued against the possibility that participants responded randomly and confirmed their feedback on task difficulties and a conservative criterion they adopted. Despite the low performance, the coherent pattern of interactions between task and level of visibility reported in the brain imaging results (see below) suggests that participants were sensitive to task demands.

\section{Imaging data}

\section{Whole brain analysis}

Unless stated otherwise, the threshold of $\mathrm{p}<.05$ with family-wise error (FWE) correction for multiple comparisons at the voxel level ( $\mathrm{t}>$ 4.62 or $\mathrm{z}>4.54$ ) was applied in the whole brain analyses presented below.

\section{Global network}

The contrasts between activation obtained on Nogo trials (all levels of visibility collapsed) and the one obtained on mask-only trials reveal the global networks of brain areas recruited during the visual, phonological and semantic tasks. As shown in Table 2 and Fig. 2a, the three tasks recruited overlapping brain areas in the left hemisphere, including the posterior regions in the inferior occipital cortex (OCC) and the fusiform gyrus (FUS), and more anterior regions in the inferior frontal gyrus pars opercularis (IFG oper) and pars triangularis (IFG tri), precentral cortex, supplementary motor area (SMA) and insula. ${ }^{2}$ Task comparisons did not show any significant result, suggesting that the same neural networks including the areas involved in orthographic, semantic, phonological and articulatory processing were activated regardless of task demand.

\footnotetext{
${ }^{2}$ We noticed some small signal losses in the anterior ventral temporal and the medial orbito-frontal regions in some participants. The artefacts in the BOLD signal in these regions are frequently observed in fMRI studies and seem to result from abrupt changes in magnetic susceptibility that occurs across tissue interfaces such as the border between brain tissue and air-filled sinuses or the auditory cavity. Although it is difficult to estimate the amount of signal attenuation in these and the surrounding areas, we could not exclude the possibility that these artefacts might somehow affect the detection of the semantic effect in our study.
}

\section{Linear effect of visibility}

The influence of stimulus driven bottom-up information was examined by searching for the areas where activation increased with the visibility of the stimulus. Based on an independent pilot study showing that even at the highest level of visibility (L3), participants' performance did not reach a plateau, we decided to fit our data with a linear function. A linear contrast with the weights $-3,-1,1,3$ for the L0, L1, L2 and L3 level of visibility, respectively, was used to estimate the slope of the BOLD response in each task. As shown in Table 2 and Fig. 2b, subsets of the areas within the global networks showed sensitivity to stimulus visibility. In the visual task, these areas were restricted to the visual cortex in both hemispheres, including the calcarine fissures and fusiform gyri. A similar activation pattern was found in the phonological task although the activation in the inferior temporal lobe at the location of the fusiform gyrus was left-lateralized. Additionally, significant linear effects were also observed in the left IFG oper, precentral cortex and SMA, i.e., areas involved in high-level phonological analyses and articulatory processing (Demonet et al., 1992; Gough et al., 2005; Poldrack et al., 1999; Price, 2012; Rumsey et al., 1997). The semantic task showed a similar activation pattern as in the phonological task, with additional activation in the left superior parietal cortex, left middle occipital lobe, bilateral thalamus and left IFG tri, i.e., a region which has also been implicated in semantic processing (Demonet et al., 1992; Gough et al., 2005; Kapur et al., 1994; Price, 2012). A negative linear contrast (Fig. 2b), reflecting a linear decrease of activation with the level of stimulus visibility, showed significant results in the left inferior parietal lobe in the semantic $(-60$ -45 42) and phonological tasks (-42 -69 42; with an uncorrected voxel-based threshold at $\mathrm{p}<.001$ combined with a cluster size FWE corrected at $\mathrm{p}<.05$ ) but not in the visual task.

It is worth noting that regional activation might increase as a function of effort, resulting in decelerating or even U-shaped effects for conditions that are increasingly easy to process. Therefore, low visibility (evidenced by low task performance, Fig. 4) could keep activation on the ascending part of the slope. To test this assumption, we examined the effects of quadratic contrast. The analysis did not reveal any significant result.

In addition to these contrasts, we also attempted to identify the areas that showed significant activation when words were presented at the lowest level of visibility (L0), that is, when they could not be identified above chance level (Fig. 4). Here, the contrasts between Nogo trials and mask-only trials did not reveal significant activation in any task.

\section{Interaction between visibility and task}

Analyses of the interactions between the positive linear effect of visibility and task indicate that, in most brain areas identified in the global networks, responses to stimulus visibility is modulated by task demand. Within the left IFG tri, IFG oper and SMA, responses to the linear contrast were significantly stronger in the semantic compared to the visual task. The interaction was restricted to the left IFG tri when the semantic was compared to the phonological task. No area showed a significant stronger linear effect when the phonological task was compared to the visual task. The visual cortex seems to be the only region that showed a constant linear effect of visibility across tasks.

The patterns of interaction between level of visibility and task were further illustrated in Fig. 3 showing the amplitude of the BOLD signals extracted from seven ROIs that were defined as intersections of spheres of $10 \mathrm{~mm}$ radius with the clusters obtained in a conjunction contrast of the three tasks at the group level (p. $<.001$, uncorrected, with a minimum of 50 contiguous voxels). These ROIs corresponded to the anterior fusiform gyrus $(-39-51-15)$, the middle fusiform gyrus ( -39 $-60-9)$, the posterior fusiform gyrus (-30 -78 -9), IFG tri (-39 12 24), precentral (-45 -3 42), SMA (-3 951 ), and insula (-30 24 3) of the left hemisphere.

In the whole brain analyses presented above, the effect of visibility was estimated by a linear contrast on the four-level visibility factor that 
Table 2

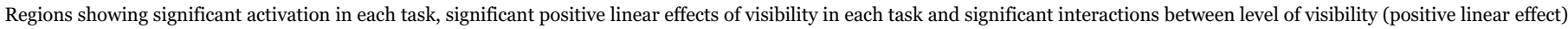
and task. Anatomical labels are obtained with the Anatomical Automatic Labeling toolbox (http://www.cyceron.fr/web/aal_anatomical_automatic_labeling.html).

$$
\text { Cluster size }
$$

$\mathrm{T}(\mathrm{df}=308)$

Z

$\mathrm{x}$

$\mathrm{y}$

Analysis at $\mathrm{p}<.05$ family-wise-error corrected (voxel-based)

Global network in the visual task

Frontal Inf Oper L
Precentral_L
Supp_Motor_Area L
Insula_L
Frontal Inf Tri L
Occipital_Inf_L
Occipital_Inf_L
Occipital_Inf_L
Caudate_R
Parietal_Inf_L

$\begin{array}{ll}279 & 7.26 \\ & 6.22 \\ 70 & 6 \\ 33 & 5.72 \\ & 4.75 \\ 97 & 7.21 \\ 26 & 5.87 \\ & 4.9 \\ 8 & 4.85 \\ 7 & 4.77\end{array}$

Global network in the phonological task

Precentral_L

Precentral_L

Cingulum_Mid_R

Supp_Motor_Area L

Insula_L

Frontal_Inf_Tri_L

Temporal_Inf_L

Temporal_Inf_L

Occipital_Mid_L

Occipital_Mid_L

Caudate_L

Caudate_R

$\begin{array}{ll}257 & 7.76 \\ & 6.6 \\ 99 & 5.92 \\ & 5.91 \\ 23 & 5.49 \\ 13 & 5.26 \\ 106 & 6.61 \\ & 5.73 \\ 56 & 5.5 \\ & 5.41 \\ 29 & 5.62 \\ 12 & 5.16\end{array}$

Global network in the semantic task

Fusiform_L

$$
\text { Fusiform_L }
$$

Occipital_Inf_L

Supp_Motor_Area_L

Precentral_L

Frontal_Inf_Tri_L

Frontal_Inf_Tri_L

$\begin{array}{ll}7.26 & 6.97 \\ 6.22 & 6.04 \\ 6 & 5.83 \\ 5.72 & 5.57 \\ 4.75 & 4.66 \\ 7.21 & 6.92 \\ 5.87 & 5.71 \\ 4.9 & 4.81 \\ 4.85 & 4.75 \\ 4.77 & 4.68\end{array}$

$\begin{array}{llll}6.97 & -42 & 12 & 21 \\ 6.04 & -42 & 0 & 42 \\ 5.83 & -6 & 9 & 51 \\ 5.57 & -30 & 24 & 3 \\ 4.66 & -39 & 24 & 12 \\ 6.92 & -42 & -60 & -12 \\ 5.71 & -27 & -78 & -9 \\ 4.81 & -36 & -81 & -9 \\ 4.75 & 12 & 12 & -3 \\ 4.68 & -24 & -48 & 51\end{array}$

Visibility effect in the visual task

7.41

6.38

5.75

5.75

5.36

5.14

6.38

5.58

5.37

5.28

5.48

5.05

6.9

5.78

4.78

6.32

5.55

5.63

5.59

5.45

$\begin{array}{lll}-39 & 6 & 30 \\ -48 & -3 & 45 \\ 9 & 15 & 42 \\ -3 & 9 & 51 \\ -30 & 27 & 0 \\ -48 & 30 & 12 \\ -42 & -60 & -9 \\ -39 & -48 & -18 \\ -24 & -51 & 42 \\ -24 & -63 & 39 \\ -9 & 9 & 0 \\ 9 & 9 & 0\end{array}$

Calcarine_R

$$
\text { Fusiform_R }
$$

Calcarine_L

Occipital_Inf_L

Occipital_Inf_L

Fusiform_L

Visibility effect in the phonological task

Frontal_Inf_Oper_L Precentral_L

Supp_Motor_Area_L Cingulum_Mid_R

Temporal_Inf_L

Calcarine_R

Calcarine_L

Occipital_Sup_L

151

48

141

6.57

5.99

6.02

5.82

5.79

5.55

Visibility effect in the semantic task

Frontal_Inf_Oper_L

Frontal_Inf_Tri_L

Insula_L

Insula_R

Supp_Motor_Area_L

Cingulum_Ant_R

Cingulum_Mid_R

Fusiform_L

Fusiform_L

Calcarine_R

Lingual_R

Fusiform_R

Fusiform_R

Occipital_Mid_L

Parietal_Sup_L

Thalamus_L

Thalamus_R
6.49

5.94

6.16

5.6

6.58

6.22

5.83

4.97

160

58

38

876

47

300

642

81

69

69
25

20

31
9.55

9.3

8.3

6.08

6.08
8.68

5.61

5.1

8.26

7.74

7.39

5.35

5.28

5.17

6.26

5.31

5.53

6.26
6.35

5.82

5.85

5.66

5.64

5.41

6.28

5.78

5.98

5.46

6.36

6.03

5.68

4.87

$>8$

$>8$
7.24

7.24

5.9

$>8$

5.47

4.99

7.83

7.39

7.09

5.22

5.16

5.06

6.07

5.19

5.39

6.07

$\begin{array}{lll}-39 & -60 & -12 \\ -36 & -51 & -15 \\ -33 & -81 & -6 \\ -3 & 9 & 51 \\ -45 & -3 & 42 \\ -39 & 21 & 21 \\ -39 & 12 & 24\end{array}$

$\begin{array}{lll}18 & -63 & 6 \\ 27 & -75 & -9 \\ -12 & -66 & 9 \\ -42 & -60 & -12 \\ -36 & -81 & -6 \\ -21 & -84 & -9\end{array}$

$\begin{array}{lll}-45 & 6 & 27 \\ -48 & 0 & 45 \\ -3 & 9 & 48 \\ 9 & 15 & 39 \\ -42 & -60 & -9 \\ 21 & -60 & 6 \\ -21 & -66 & 6 \\ -24 & -69 & 27\end{array}$

$\begin{array}{lll}-39 & 9 & 27 \\ -48 & 30 & 12 \\ -30 & 24 & 0 \\ 33 & 24 & 3 \\ -3 & 9 & 51 \\ 6 & 9 & 27 \\ 6 & 27 & 30 \\ -42 & -54 & -12 \\ -36 & -51 & -18 \\ 15 & -66 & 9 \\ 18 & -84 & -6 \\ 30 & -72 & -9 \\ 36 & -54 & -15 \\ -27 & -72 & 27 \\ -24 & -54 & 45 \\ -9 & -15 & 9 \\ 9 & -12 & 3 \\ & \text { (continued on next page) }\end{array}$




\section{Cluster size}

$\mathrm{T}(\mathrm{df}=308)$
NS

$\begin{array}{lll}62 & 5.41 \\ & 5 & 5.11 \\ & & 4.81 \\ & & 4.77\end{array}$

Analysis at $\mathbf{p}<.05$ family-wise-error corrected (voxel-based) Global network in the visual task

\section{Visibility * Task interaction}

Semantic $>$ Visual

Frontal_Inf_Tri_L

Frontal_Inf_Oper_L

Supp_Motor_Area_R

Semantic $>$ Phonological

Frontal_Inf_Tri_L

Phonological $>$ Visual
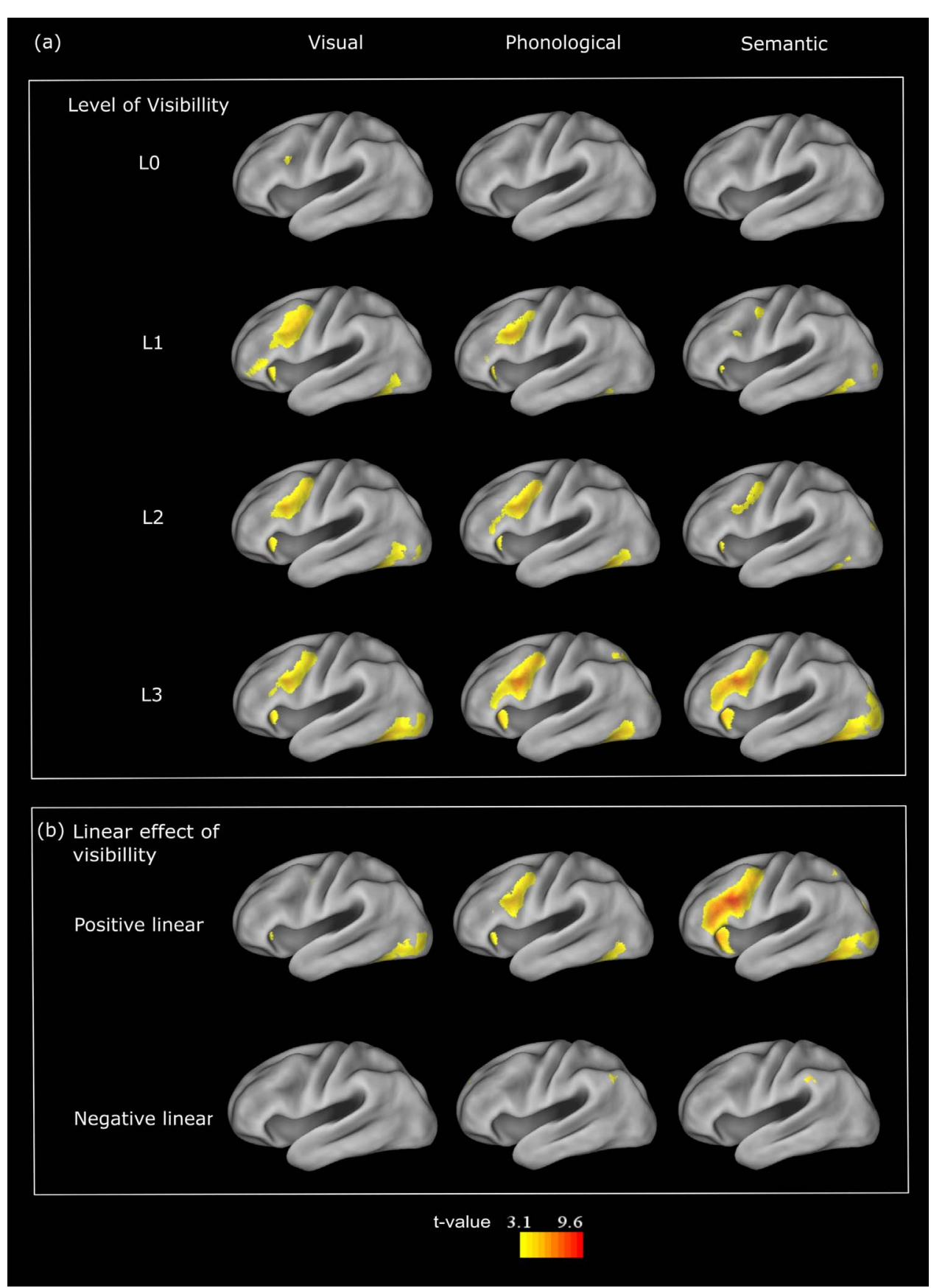

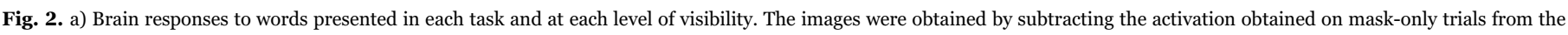

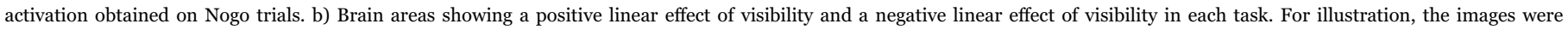
thresholded at $\mathrm{p}<.001$, uncorrected. 


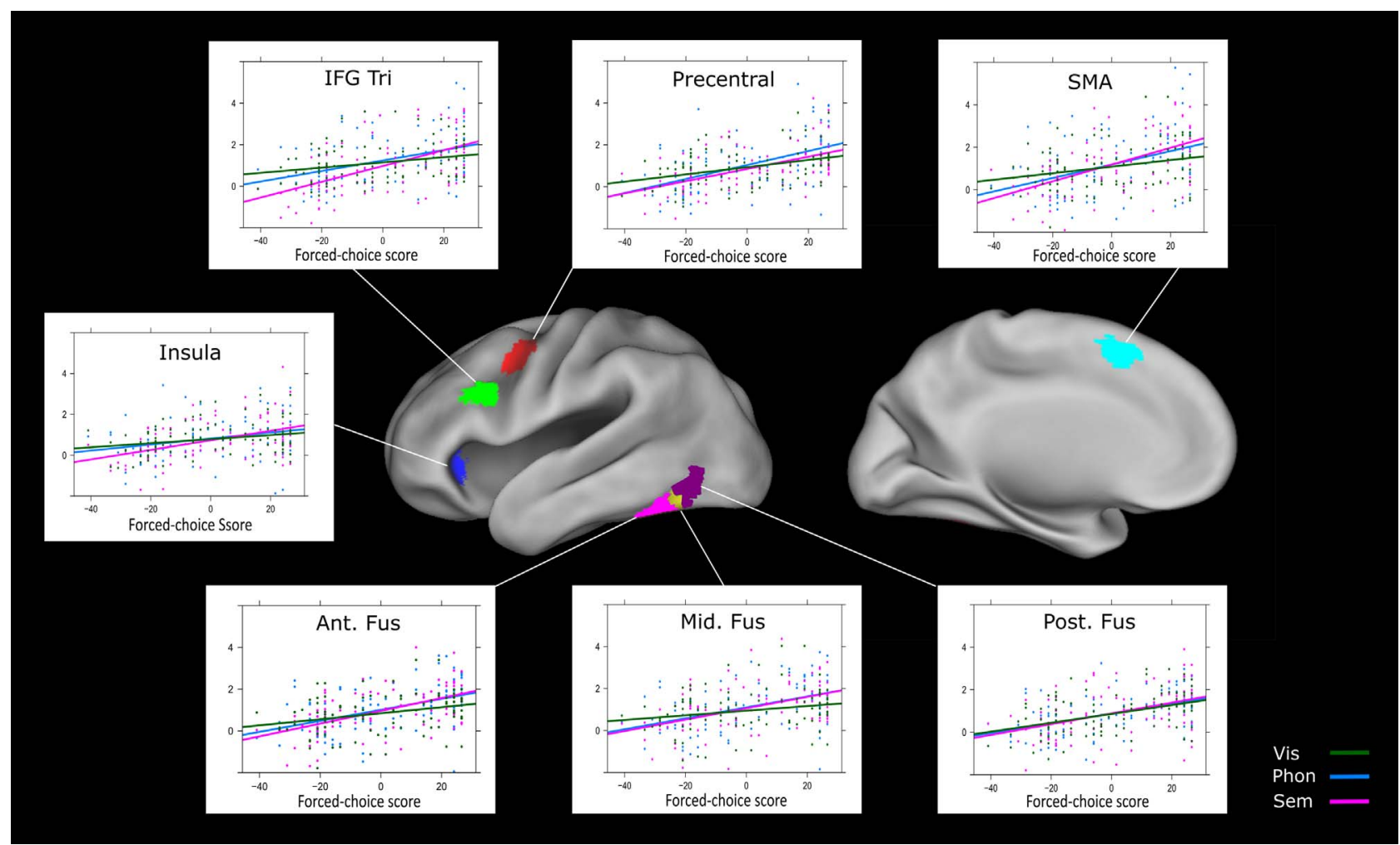



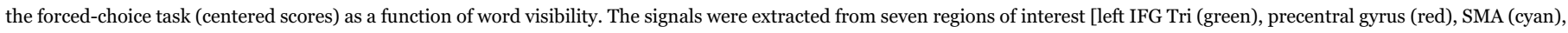



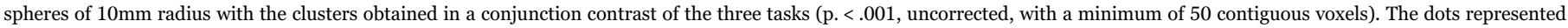
individual data. The lines represented the fit by linear regressions.

was experimentally manipulated. However, it is possible that there are strong inter-individual differences in participants' sensitivity to the visual input. In the regions of interest analyses, we replaced this linear factor by the subject-specific visibility estimated during the forcedchoice task that better reflects the actual level of visibility for the participant. The average BOLD signal in the ROIs were analyzed with mixed-effect general linear models (one per ROI), with task and individual scores obtained in the forced-choice $\operatorname{task}^{3}$ (centered scores, Fig. 4b) as fixed factors and subjects as a random-factor. The findings confirmed the patterns of interaction reported in Table 2. As shown in Table 3 and Fig. 3, significant interactions between level of visibility and task were detected in most ROIs. Precisely, in the left anterior and middle fusiform gyrus (at the location of the Visual Word Form Area reported in previous studies, e.g., Cohen et al., 2002; Jobard et al., 2003; Binder et al., 2006), precentral gyrus, SMA and IFG tri, the effect of stimulus visibility was significantly (or marginally, $\mathrm{p} \leq .08$ ) stronger in both semantic and phonological tasks compared to visual task. In the left insula, the interaction reflects a stronger effect of stimulus visibility in the semantic compared with visual task. Finally, in the IFG tri, sensitivity to stimulus visibility was marginally stronger in the semantic compared to phonological task. The left posterior fusiform gyrus

\footnotetext{
${ }^{3}$ ANOVA taking into account level of visibility and type of distractor (no overlap vs. partial overlap) showed significant effects of level of visibility $[F(3,66)=128, p<.001]$ and type of distractor $[F(1,22)=9.3, p=.005]$. However, the interaction between the two factors was not significant $[F(3,66)=1.9, p>.10]$. As shown in Fig. $4 \mathrm{a}$, on both types of trial, participants' performance at L0, i.e., the lowest level of visibility, was not different from chance level ( $p$ s $>.40$ ). Their average success rates were above chance at L1, L2 and L3 ( $p s \leq .001$ ). In the ROI analyses using mixed-effect models, the scores from both types of distractor were collapsed in order to obtain a unique score and to increase the number of observations per level of visibility. However, we also ascertained that the mixed-effect analyses performed on the scores on no overlap and partial overlap trials led to the same conclusion.
}

was the only area where the response to stimulus visibility was not affected by task demands. ${ }^{4}$

\section{Brain activation within the spoken language system: Subject-specific ROI analyses}

In the whole-brain analyses, the areas that showed significant activation in response to written words were mainly located in the prefrontal and occipito-temporal lobes. Yet, given the existence of functional and anatomical connections between the visual and auditory systems (Booth et al., 2002; Thiebaut De Schotten et al., 2014; Vagharchakian et al., 2012; Yeatman et al., 2011), it is likely that processing written words also induces activation in the spoken language system, although to a lesser extent. To further examine how the spoken language system is influenced by written words' visibility and task demand, we restricted our analyses to the cortical areas that process acoustic, phonological and semantic contents of spoken sentences, using the subject-specific approach proposed by NietoCastañón and Fedorenko (2012). The same contrasts of interest as in the whole brain analyses (cf. Table 2) were examined with a threshold of $\mathrm{p}<.05$ corrected for multiple comparisons across the ROIs using the False Detection Rate method (FDR) as proposed in the spm_ss toolbox (http://www.nitrc.org/projects/spm_ss). Overall, the pattern of activation obtained in the temporal areas identified by the auditory localizers differed from the main pattern found in the written language

\footnotetext{
${ }^{4}$ Similar analyses conducted with visibility as a four-level factor instead of forcedchoice score and looking at the linear effect of visibility, yielded similar conclusions. In some brain areas (i.e., anterior, middle and posterior parts of the left fusiform gyrus and in the precentral cortex) a quadratic function could also explain the variation of the BOLD signal. However, its contribution was only marginal ( $p$ values varied from 0.04 to 0.08 ) and much weaker than that of the linear function ( $p$ values varied from 0.004 to $<.0001)$.
} 
(a)

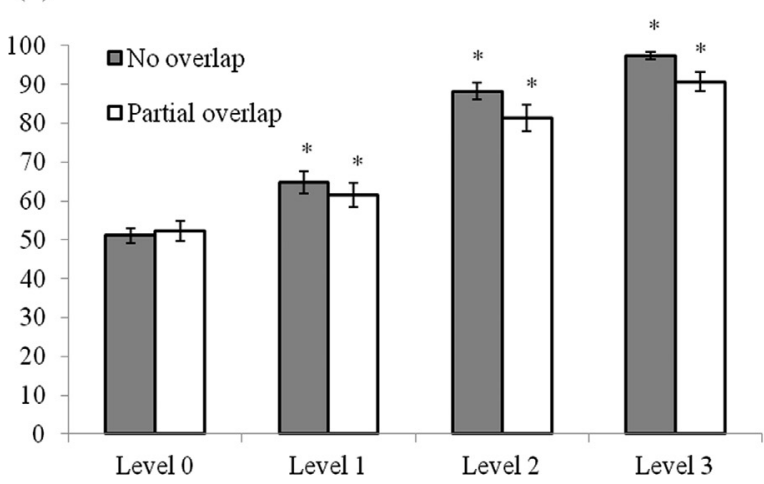

(b)

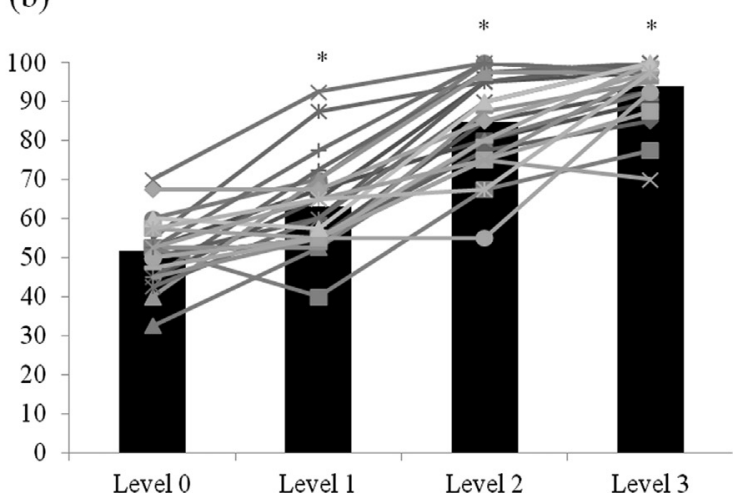

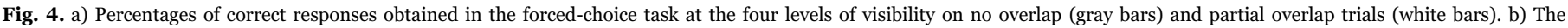

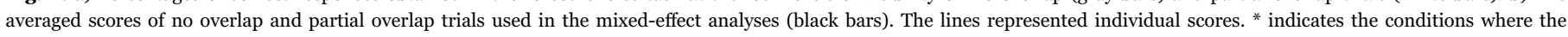
accuracy scores differed from the $50 \%$ value expected by chance.

Table 3

Results of the mixed effect analyses testing the interaction effects between stimulus visibility and task on the amplitude of the BOLD signals extracted from the seven ROIs. The p. values derived from the lmerTest package (Kuznetsova et al., 2013).

\begin{tabular}{|c|c|c|c|c|c|c|}
\hline ROIs & Visibility*Task & Estimate & $\mathrm{SE}$ & $\mathrm{df}$ & t. value & p. value \\
\hline \multirow{3}{*}{ Ant. Fus } & Vis vs. Phon & 0.012 & 0.0066 & 248 & 1.812 & .07 \\
\hline & Vis vs. Sem & 0.016 & 0.0066 & 248 & 2.441 & .015 \\
\hline & Phon vs. Sem & 0.004 & 0.0066 & 248 & 0.630 & .53 \\
\hline \multirow{3}{*}{ Mid. Fus } & Vis vs. Phon & 0.015 & 0.0072 & 248 & 2.091 & .04 \\
\hline & Vis vs. Sem & 0.016 & 0.0072 & 248 & 2.245 & .03 \\
\hline & Phon vs. Sem & 0.001 & 0.0072 & 248 & 0.154 & .88 \\
\hline \multirow{3}{*}{ Post. Fus } & Vis vs. Phon & 0.002 & 0.0067 & 248 & 0.312 & .76 \\
\hline & Vis vs. Sem & 0.004 & 0.0067 & 248 & 0.668 & .51 \\
\hline & Phon vs. Sem & 0.002 & 0.0067 & 248 & 0.356 & .72 \\
\hline \multirow{3}{*}{ IFG Tri } & Vis vs. Phon & 0.01 & 0.0071 & 248 & 1.787 & .08 \\
\hline & Vis vs. Sem & 0.02 & 0.0071 & 248 & 3.559 & .0004 \\
\hline & Phon vs. Sem & 0.01 & 0.0071 & 248 & 1.772 & .08 \\
\hline \multirow{3}{*}{ Precentral } & Vis vs. Phon & 0.02 & 0.0063 & 248 & 2.568 & .01 \\
\hline & Vis vs. Sem & 0.01 & 0.0063 & 248 & 1.891 & .06 \\
\hline & Phon vs. Sem & -0.004 & 0.0063 & 248 & -0.676 & .50 \\
\hline \multirow{3}{*}{ SMA } & Vis vs. Phon & 0.02 & 0.0082 & 248 & 1.968 & .05 \\
\hline & Vis vs. Sem & 0.02 & 0.0082 & 248 & 2.916 & .004 \\
\hline & Phon vs. Sem & 0.008 & 0.0082 & 248 & 0.947 & .34 \\
\hline \multirow{3}{*}{ Insula } & Vis vs. Phon & 0.005 & 0.0057 & 248 & 0.825 & .41 \\
\hline & Vis vs. Sem & 0.01 & 0.0057 & 248 & 2.365 & .02 \\
\hline & Phon vs. Sem & 0.009 & 0.0057 & 248 & 1.541 & .12 \\
\hline
\end{tabular}

network. As shown in Fig. 5 (lower panel), the "acoustic" localizer (scrambled sentences > silent baseline contrast) activated three ROIs within the bilateral superior temporal gyri (STG), including the Heschl gyri (MNI peak coordinates: $54-206 ;-40-3312 ;-53-206$ ). None of these areas responded to written words or were sensitive to stimulus visibility or its interaction with task $(\mathrm{p}>.50)$. The "phonological" localizer (pseudo sentences > scrambled sentences contrast) activated four ROIs within the bilateral STG (-58 -12 0; $60-63 ; 58$ $-250 ;-61-323)$. When all levels of visibility were collapsed, the left STG (-61 -32 3, Fig. 5, in green) showed significant activation in the visual $\left(\mathrm{p}=.05\right.$, effect size $\left.^{5}=0.7\right)$ and phonological $(\mathrm{p}=.06$, effect size $=0$. 7) but not in semantic task $(\mathrm{p}=.11$, effect size $=0.6)$. However, in the three tasks, the response within this area increased linearly with

\footnotetext{
${ }^{5}$ The effect sizes reported in the subject-specific ROI analyses correspond to percent BOLD signal change relative to either the mask-only baseline or other experiment conditions (when different conditions were compared).
}

stimulus visibility ( $\mathrm{p} .=.001$, effect $\operatorname{size}=4.24 ; \mathrm{p}=.03$, effect size $=3.56$; $\mathrm{p}=.02$, effect size $=3.37$ for the visual, phonological and semantic task, respectively). Unlike the typical profile obtained in the written language network, the sensitivity of the left STG to stimulus visibility was not significantly modulated by task demands (interactions between task demands and visibility; $p s>.70$ ). No significant result was observed in the other phonological ROIs. Finally, the "semantic" localizer (real sentences > pseudo sentences contrast) led to significant activation in only one area in the left anterior middle temporal gyrus (-55 -11 -13, Fig. 5, in purple). When all levels of visibility were collapsed, the area was significantly activated only in the semantic task ( $p=.04$, effect size $=0.524$ ). Its response increased linearly with the level of visibility only when participants performed the phonological task $(\mathrm{p}=.01$, effect size $=2.46$ ), although the interactions between visibility and task did not reach significance $(p s>.10)$.

The results from mixed effects analyses (with task and individual responses to stimulus visibility obtained in the forced-choice task as fixed factors and subject as a random factor) performed on the percent BOLD signal change (relative to the mask-only trials) of the individually defined fROIs extracted from the subject-specific ROI analyses (Nieto-Castañón and Fedorenko, 2012) only showed significant result in the left STG identified by the phonological localizer (-61 -32 3, Fig. 5, in green). As illustrated in the upper panel of Fig. 5, in the three tasks, the BOLD signals significantly increased with participants' performance in the forced-choice task [visual task: estimate $=0.02$, $\mathrm{SE}=0.007, \mathrm{t}(151)=2.97, \mathrm{p}=.003$; phonological task: estimate $=0.02$, $\mathrm{SE}=0.007, \mathrm{t}(151)=2.85, \mathrm{p}=.005 ;$ semantic task: estimate $=0.02$, $\mathrm{SE}=0.007, \mathrm{t}(151)=2.76, \mathrm{p}=.007]$. No interaction between task and level of visibility was observed. The models failed to detect significant effects in the other ROIs.

\section{Discussion}

Written words presented during symbol detection, rime detection and semantic judgment tasks elicited activity in the same left-hemisphere networks including the posterior regions involved in visual (inferior occipital cortex) and higher-level orthographic processing (fusiform gyrus) and more anterior regions involved in semantic (IFG tri), phonological (IFG oper) and articulatory processing (precentral cortex, SMA, insula) among other functions. Note however that the absence of the BOLD signal in the anterior ventral temporal regions that are typically involved in semantic processing could be due to signal dropout frequently observed across tissue interfaces such as the border between brain tissue and air-filled sinuses or the auditory cavity (Devlin et al., 2000). Interestingly, written words also elicited activity in the left temporal cortex, in the vicinity of the superior temporal gyrus and the anterior middle temporal gyrus. These areas corre- 


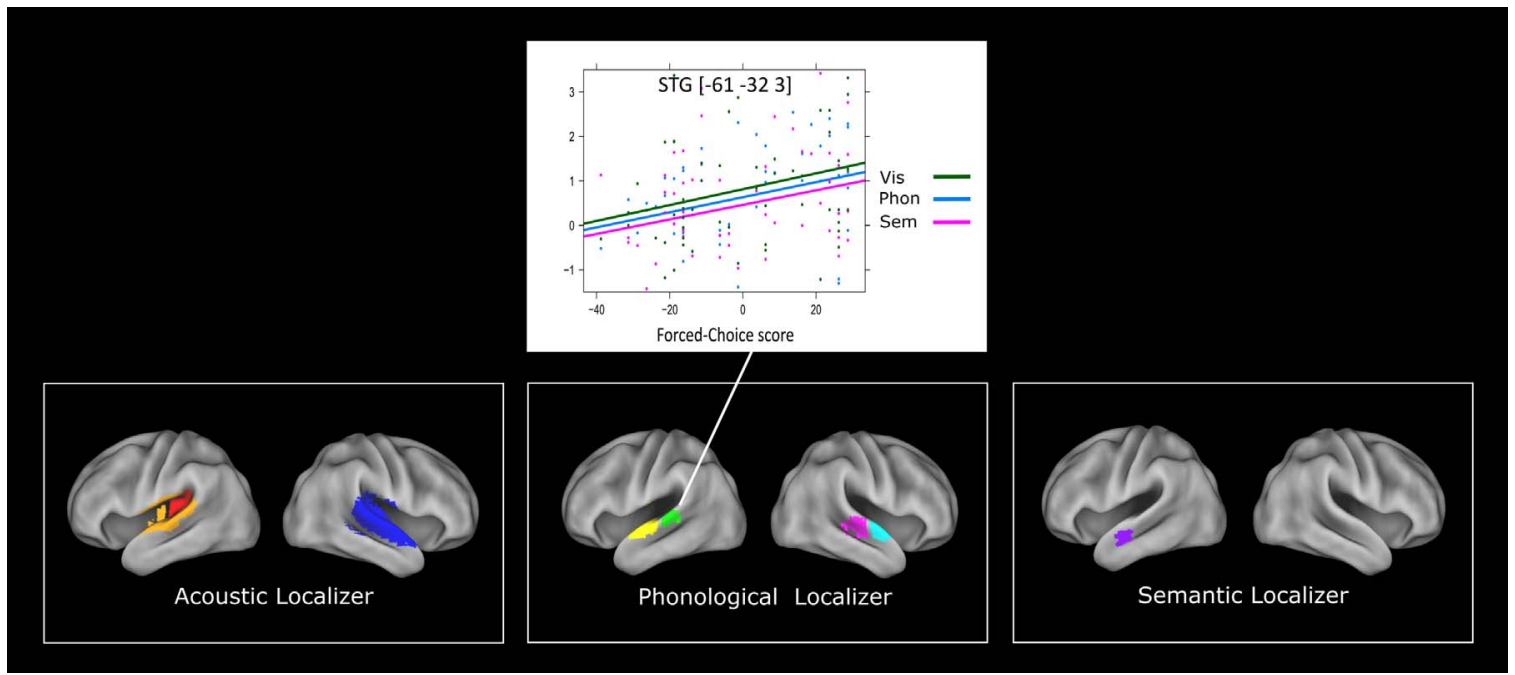



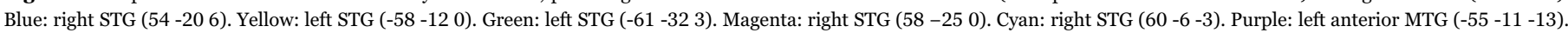

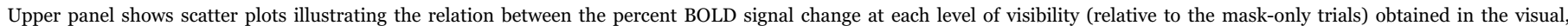

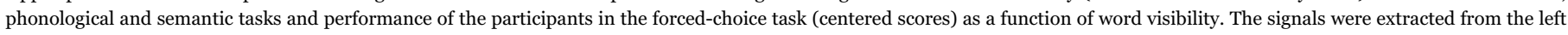
superior temporal gyrus (-61 -32 3) identified by the phonological localizer. The dots represented individual data. The lines represented the fit by linear regressions.

sponded to the ones involved in phonological and semantic processing of the spoken sentences presented in our auditory localizer tasks (see also Binder et al., 2009, 1994; Demonet et al., 1992; Price, 2012). However, the activation in these temporal regions was somewhat weaker than in the posterior and anterior regions since it was significant only in the subject-specific ROI analysis.

\section{Stimulus-driven activation of orthographic, phonological and semantic processes}

Previous studies reported that neural responses of only a few areas within the occipito-temporal cortex increased linearly with visibility or presentation duration of written input (Ben-Shachar et al., 2007; Price and Friston, 1997; Vagharchakian et al., 2012). Here, we found a linear activation pattern in all of the areas identified in the network described above. However, it is likely that this widespread linear trend is specific to the very early stage of written word processing investigated here. Price and Friston (1997) manipulated the presentation duration of written words in a larger time-window (from 150 to $1250 \mathrm{~ms}$ ) when their participants performed silent reading and reading aloud tasks. The authors found linear monotonic increases of activation with stimulus duration only in visual processing areas. In higher-order language areas, the neural responses progressed linearly at least until 400-600 ms where activation reached a plateau and decreased afterwards.

As illustrated in Fig. 2, the passage from the lowest level of visibility (L0, where words could not be identified above chance level) to the next level (L1, where written words could be identified above chance level, cf. Fig. 4) was associated with an increase of activation within the visual system and a spread of activation to more distant anterior regions. This activation pattern is coherent with findings from subliminal vs. conscious perception studies. As reviewed by Dehaene et al. (2006), the passage from subliminal to conscious perception of visual stimulus is indicated by an amplification of occipito-temporal activation with extension of brain activation to higher association cortices interconnected via long-distance connections. In the present study, such extension of brain activation suggests that the presence of written words automatically triggers activity in the areas that process highlevel orthographic, phonological and semantic representations from the moment words are consciously perceived. Interestingly, this linear increase of activation in the left posterior and anterior brain regions was also coupled with a linear decrease of activation in the left inferior parietal lobe, especially in the semantic and phonological tasks. The localization of this negative linear response indicates a deactivation of the default mode network when greater effort is needed to actively extract phonological and semantic information from visual input (Boros et al., 2016; Mason et al., 2007; Raichle et al., 2001).

\section{Task-driven activation of orthographic, phonological and semantic} processes

When all levels of visibility were collapsed, task comparisons did not reveal any significant difference in the reading network. At first glance, the absence of the task effect seems to suggest that a written word triggers orthographic, phonological and semantic processes in the same way regardless of task demands. However, analyses of the interactions between stimulus visibility and task changed the picture. Indeed, we found two subsets of brain areas that varied as a function of their sensitivity to task demands. The first subset regroups high-order language areas that showed significant interactions between stimulus visibility and task. Interestingly, the interaction pattern depended on the functional role of each area in a specific task set, such that, in response to the same sensory input, top-down process enhanced neural responses in the areas that were directly relevant to the task. Precisely, in most areas identified in the network, including the left IFG pars opercularis, IFG pars triangularis, precentral, SMA, and the anterior and middle parts of the fusiform gyrus, i.e., the areas assumed to be involved in high-level language functions, stronger responses to stimulus visibility were found in the semantic and, to a lesser extent, phonological tasks compared to the visual task. Importantly, the fact that the anterior and middle parts of the FUS and the prefrontal cortex showed a similar pattern of activation is coherent with previous studies showing intrinsic functional connections that link the ventral occipitotemporal cortex (particularly at the site of the Visual Word Form Area) with the IFG (Bitan et al., 2005; Koyama et al., 2010). However, further studies using techniques with higher temporal resolution are needed to decide whether the task-related effect that was observed in the occipitotemporal orthographic system occurred within the system itself or resulted from top-down projections from prefrontal regions as is typically assumed (Gilbert and Sigman, 2007; Price and Devlin, 2011; Twomey et al., 2011; Woodhead et al., 2014).

In addition to this main activation pattern, we also found an 
increase of sensitivity to the sensory input in the IFG par triangularis when the semantic task was compared to the phonological task and in the anterior insula when it was compared to the visual task. The anterior insula showed significant activation in most linguistic tasks and is assumed to play a role in subvocal rehearsal during both language production and language perception (Riecker et al., 2000; Wise et al., 1999). The fact that it showed a stronger response to stimulus visibility in the semantic compared to visual but not to phonological task might reflect an equally strong engagement of phonological and sub-articulatory processes during the semantic and phonological tasks. This observation will be discussed further below.

The second subset of brain areas showed significant responses to stimulus sensitivity without an interaction with task. This purely stimulus-driven activation was restricted to the posterior FUS and the STG. The disparity between the activation patterns obtained in the posterior FUS and in the anterior and middle FUS supports a functional distinction between the different parts of the structure, with the posterior part being involved in low-level visual or sublexical process and the middle and anterior parts in lexico-semantic process (Ludersdorfer et al., 2015; Price and Mechelli, 2005; Vinckier et al., 2007). Finally, the dissociation between the activation patterns observed in phonological areas located in the prefrontal cortex (IFG oper) and the temporal cortex (STG) corroborates some previous studies on spoken language processing showing differences in the sensitivity to top-down vs. bottom-up process in these two regions (Davis et al., 2011; Zekveld et al., 2006). Thus, the conclusion obtained in the studies using auditory stimuli remains valid even when the activation of the auditory system is elicited by visual input. Although this specific activation profile obtain in the post FUS and STG is more compatible with the idea that top-down information does not contribute to information processing in lower-level language areas, the current data set does not allow us to rule out the possibility that the activation profile may evolve over time (Price and Friston, 1997) and the impact of task demands may be delayed and become detectable only later on.

Mechanisms involved in the activation of phonological and semantic representations during written word processing

Previous studies provided evidence in favor of both mandatory and task-dependent activations of phonological and semantic information during reading. Our findings are compatible with both observations. On the one hand, written words elicited activation in areas associated with semantic, phonological and subvocal articulatory processes in a taskindependent manner, as illustrated by significant activations of these areas not only in phonological and semantic tasks but also in a low-level visual task. This observation is predicted by connectionist models that assume an automatic spread of activation from the visual to the phonological and semantic systems (Harm and Seidenberg, 2004, 1999; Seidenberg and McClelland, 1989). On the other hand, the automatic account could not explain the entire set of findings since task demands also modulate neural responses in high-level language areas by enhancing their sensitivity to the sensory input in a task-specific manner.

Price et al. (1997) made a distinction between implicit (unconscious) and explicit (attentional and task-dependent) activation of phonological and semantic representations during written word processing. In the present study, the implicit mechanism could indeed explain activation observed in the areas that are not relevant to the task. This was the case for the activation of high-level orthographic, semantic, phonological and articulatory areas during the low-level visual task, the activation of semantic areas during the phonological task, the activation of phonological and articulatory areas during the semantic task and, finally, the activation of the auditory cortex in all tasks. By contrast, the explicit mechanism provides an explanation for enhancements of sensitivity to the visual input observed in the anterior and middle FUS and the prefrontal areas when participants performed the semantic and phonological compared to low-level visual tasks, in the IFG pars triangularis when they performed the semantic compared to the phonological task and in the insula when they performed the semantic compared to visual task. Human and primate imaging data showed top-down enhancement of neural processing of relevant sensory input and reduction of neural sensitivity to noise or distractors (Gilbert and Sigman, 2007; Kastner and Ungerleider, 2000; Kiefer, 2007; Sarter et al., 2001). Our findings complement these observations by showing that such enhancement of neural responses also applies to abstract, phonological and semantic, representations associated with written words. The findings provide the neurobiological basis for some behavioral findings. For instance, Kiefer (2007) reported masked semantic priming only when the preceding task set required focusing on semantic word features, but not when it required focusing on perceptual word features. Such semantic priming effect might be underpinned by a task-specific enhancement of sensitivity of the neurons in the semantic areas in responses to (or perhaps even before the presence of) visual input.

\section{Stronger association between orthography and phonology than between orthography and semantics}

Another finding of interest was that, across different analyses, the areas usually considered to be involved in phonological and articulatory processes, like IFG pars opercularis, precentral and SMA, equally responded in both semantic and phonological tasks despite the fact that neither phonological analysis nor speech production was required in the semantic task. On the contrary, the IFG pars triangularis, involved in semantic process, showed stronger responses to stimulus visibility in the semantic compared to phonological task. Thus, it seems that while the activation of word pronunciation and the corresponding subvocal articulatory process occurs in both phonological and semantic tasks, the activation of word meaning may be more task-specific. Prior studies already provided evidence in favor of a task-specific activation of the anterior part of the IFG: While this area tends to be more strongly activated in semantic compared to phonological tasks, the posterior IFG tends to be equally activated in both tasks (Buckner et al., 1995; Poldrack et al., 1999; Wagner et al., 2000). This asymmetry in the activation of phonological/articulatory vs. semantic representations from written input has frequently been explained by the assumption that semantic processing tasks typically require access to both semantic and phonological stimulus attributes or that access to meaning is performed through the activation of phonological information (Coltheart et al., 1994; Lesch and Pollatsek, 1993; Price et al., 1997). However, this may not be the only reasons. Learning to read in alphabetic languages establishes strong connections between written and spoken codes (Ziegler et al., 2014). It has been argued that the connections between spelling and sound are stronger and more automatic than the connections between spelling and meaning because the former are highly systematic in alphabetic writing systems while the latter are mostly arbitrary (i.e., knowing that a word starts with the letter B does not tell us anything about its meaning). This could provide an alternative explanation to the asymmetric activation of phonological and semantic representations during reading.

In conclusion, the current findings suggest that the contribution of phonological and semantic processes in reading is supported by two complementary mechanisms. The first mechanism, operating in a taskindependent manner, results from an automatic spread of activation throughout a network in which orthography is linked to phonology and semantics (Harm and Seidenberg, 2004, 1999; Seidenberg and McClelland, 1989). The second, task-dependent mechanism exerts its influence by tuning the sensitivity of high-level language areas to the sensory input in a task-dependent manner. The variation of activation patterns across brain areas observed in the present study reflects an interplay between the two mechanisms during written word processing and provides a coherent explanation to seemingly inconsistent findings in the literature. 


\section{Acknowledgements}

This work was supported by the French Ministry of Research. Grant numbers: ANR-13-JSH2-0002 to C.P., ANR-11-LABX-0036 and ANR11-IDEX-0001-02. We also thank Aurélie Ponz and Virginie Epting for running the experiment and Evelina Fedorenko for insightful discussions on the subject-specific ROI analyses.

\section{References}

Belin, P., Zatorre, R.J., Ahad, P., 2002. Human temporal-lobe response to vocal sounds. Cogn. Brain Res 13, 17-26. http://dx.doi.org/10.1016/S0926-6410(01)00084-2.

Ben-Shachar, M., Dougherty, R.F., Deutsch, G.K., Wandell, B.A., 2007. Differential sensitivity to words and shapes in ventral occipito-temporal cortex. Cereb. Cortex 17, 1604-1611.

Besner, D., Stolz, J. a, Boutilier, C., 1997. The stroop effect and the myth of automaticity. Psychon. Bull. Rev. 4, 221-225. http://dx.doi.org/10.3758/BF03209396.

Binder, J.R., Desai, R.H., Graves, W.W., Conant, L.L., 2009. Where is the semantic system? A critical review and meta-analysis of 120 functional neuroimaging studies. Cereb. Cortex 120, 2767-2796.

Binder, J.R., Medler, D.A., Westbury, C.F., Liebenthal, E., Buchanan, L., 2006. Tuning of the human left fusiform gyrus to sublexical orthographic structure. Neuroimage 33, $739-748$

Binder, J.R., Rao, S.M., Hammeke, T.A., Yetkin, F.Z., Jesmanowicz, A., Bandettini, P.A., Wong, E.C., Estkowski, L.D., Goldstein, M.D., Haughton, V.M., 1994. Functional magnetic resonance imaging of human auditory cortex. Ann. Neurol. 35, 662-672.

Bitan, T., Booth, J.R., Choy, J., Burman, D.D. Gitelman, D.R., Mesulam, M.M., 2005. Shifts of effective connectivity within a language network during rhyming and spelling. J. Neurosci. 25, 5397-5403.

Booth, J.R., Burman, D.D., Meyer, J.R., Gitelman, D.R., Parrish, T.B., Mesulam, M.M. 2002. Functional anatomy of intra-and cross-modal lexical tasks. Neuroimage 16, $7-22$.

Boros, M., Anton, J.L., Pech-Georgel, C., Grainger, J., Szwed, M., Ziegler, J.C., 2016. Orthographic processing deficits in developmental dyslexia: beyond the ventral visual stream. Neuroimage 128, 316-327. http://dx.doi.org/10.1016/ j.neuroimage.2016.01.014

Brown, M.S., Roberts, M.A., Besner, D., 2001. Semantic processing in visual word recognition: activation blocking and domain specificity. Psychon. Bull. Rev. 8, $778-784$.

Brysbaert, M., 2001. Prelexical phonological coding of visual words in Dutch: automatic after all. Mem. Cogn. 29, 765-773. http://dx.doi.org/10.3758/BF03200479.

Brysbaert, M., Van Dyck, G., Van de Poel, M., 1999. Visual word recognition in bilinguals: evidence from masked phonological priming. J. Exp. Psychol. Hum. Percept. Perform. 25, 137-148.

Buckner, R.L., Raichle, M.E., Petersen, S.E., 1995. Dissociation of human prefrontal cortical areas across different speech production tasks and gender groups. J. Neurophysiol. 74, 2163-2173.

Carreiras, M., Mechelli, A., Estévez, A., Price, C.J., 2007. Brain activation for lexical decision and reading aloud: two sides of the same coin? J. Cogn. Neurosci. 19, 433-444.

Cohen, L., Lehericy, S., Chochon, F., Lemer, C., Rivaud, S., Dehaene, S., 2002. Languagespecific tuning of visual cortex? Functional properties of the visual word form area. Brain 125, 1054-1069.

Coltheart, V., Patterson, K., Leahy, J., 1994. When a ROWS is a ROSE: phonological effects in written word comprehension. Q. J. Exp. Psychol. Sect. A 47, 917-955.

Davis, M.H., Ford, M.A., Kherif, F., Johnsrude, I.S., 2011. Does semantic context benefit speech understanding through "top-down" processes? Evidence from time-resolved sparse fMRI. J. Cogn. Neurosci. 23, 3914-3932.

Deacon, D., Hewitt, S., Yang, C.M., Nagata, M., 2000. Event-related potential indices of semantic priming using masked and unmasked words: evidence that the N400 does not reflect a post-lexical process. Cogn. Brain Res. 9, 137-146.

Dehaene, S., Changeux, J.-P., Naccache, L., Sackur, J., Sergent, C., 2006. Conscious, preconscious, and subliminal processing: a testable taxonomy. Trends Cogn. Sci. 10, 204-211.

Dehaene, S., Naccache, L., Cohen, L., Bihan, D.L., Mangin, J.F., Poline, J.B., Rivière, D., 2001. Cerebral mechanisms of word masking and unconscious repetition priming. Nat. Neurosci. 4, 752-758.

Demonet, J., Chollet, F., Ramsay, S., Cardebat, D., Nespoulous, J.L., Wise, R., Rascol, A., Frackowiak, R., 1992. The anatomy of phonological and semantic processing in normal subjects. Brain 115, 1753-1768.

Devlin, J.T., Matthews, P.M., Rushworth, M.F.S., 2003. Semantic processing in the left inferior prefrontal cortex: a combined functional magnetic resonance imaging and transcranial magnetic stimulation study. J. Cogn. Neurosci. 15, 71-84. http:// dx.doi.org/10.1162/089892903321107837.

Devlin, J.T., Russell, R.P., Davis, M.H., Price, C.J., Wilson, J., Moss, H.E., Matthews, P.M., Tyler, L.K., 2000. Susceptibility-induced loss of signal: comparing PET and fMRI on a semantic task. Neuroimage 11, 589-600. http://dx.doi.org/10.1006/ nimg.2000.0595.

Drieghe, D., Brysbaert, M., 2002. Strategic effects in associative priming with words, homophones, and pseudohomophones. J. Exp. Psychol. Learn. Mem. Cogn. 28, 951-961. http://dx.doi.org/10.1002/eji.201444988.This.

Fedorenko, E., Hsieh, P.J., Nieto-Castañón, A., Whitfield-Gabrieli, S., Kanwisher, N., 2010. New method for fMRI investigations of language: defining ROIs functionally in individual subjects. J. Neurophysiol. 104, 1177-1194.

Ferrand, L., Grainger, J., 1994. Effects of orthography are independent of phonology in masked form priming. Q. J. Exp. Psychol. 47, 365-382.

Frost, R., 1998. Toward a strong phonological theory of visual word recognition: true issues and false trails. Psychol. Bull. 123, 71-99.

Gilbert, C.D., Sigman, M., 2007. Brain States: top-down Influences in Sensory Processing. Neuron 54, 677-696.

Gough, P.M., Nobre, A.C., Devlin, J.T., 2005. Dissociating linguistic processes in the left inferior frontal cortex with transcranial magnetic stimulation. J. Neurosci. 25, 8010-8016.

Harm, M.W., Seidenberg, M.S., 2004. Computing the meanings of words in reading: cooperative division of labor between visual and phonological processes. Psychol. Rev. 111, 662-720.

Harm, M.W., Seidenberg, M.S., 1999. Phonology, reading acquisition, and dyslexia: insights from connectionist models. Psychol. Rev. 106, 491-528.

Henson, R.N., 2015. Design efficiency. In: Toga, A.W. (Ed.), Brain Mapping: An Encyclopedic Reference. Academic Press: Elsevier, 489-494.

Jobard, G., Crivello, F., Tzourio-Mazoyer, N., 2003. Evaluation of the dual route theory of reading: a metanalysis of 35 neuroimaging studies. Neuroimage 20, 693-712.

Kapur, S., Rose, R., Liddle, P.F., Zipursky, R.B., Brown, G.M., Stuss, D., Houle, S., Tulving, E., 1994. The role of the left prefrontal cortex in verbal processing: semantic processing or willed action? Neuroreport 5, 2193-2196.

Kastner, S., Ungerleider, L.G., 2000. Mechanisms of visual attention in the human cortex. Annu. Rev. Neurosci. 23, 315-341.

Kiefer, M., 2007. Top-down modulation of unconscious' automatic'processes: a gating framework. Adv. Cogn. Psychol. 3, 289-306.

Kiefer, M., Martens, U., 2010. Attentional sensitization of unconscious cognition: task sets modulate subsequent masked semantic priming. J. Exp. Psychol. Gen. 139, 464-489.

Kiefer, M., Spitzer, M., 2000. Time course of conscious and unconscious semantic brain activation. Neuroreport 11, 2401-2407.

Kouider, S., Dehaene, S., Jobert, A., Le Bihan, D., 2007. Cerebral bases of subliminal and supraliminal priming during reading. Cereb. Cortex 17, 2019-2029.

Koyama, M.S., Kelly, C., Shehzad, Z., Penesetti, D., Castellanos, F.X., Milham, M.P., 2010. Reading networks at rest. Cereb. Cortex, 2549-2559.

Kuznetsova, A., Brockhoff, P.B., Christensen, R.T., 2013. LmerTest: tests for random and fixed effects for linear mixed effect models (lmer objects of lme4 package). R package version 2.0-3. 〈http://CRAN.R-project.org/package=lmerTest

Labuschagne, E.M., Besner, D., 2015. Automaticity revisited: when print doesn't activate semantics. Front. Psychol. 6.

Lesch, M.F., Pollatsek, A., 1993. Automatic access of semantic information by phonological codes in visual word recognition. J. Exp. Psychol. Learn. Mem. Cogn. 19, 285-294.

Ludersdorfer, P., Kronbichler, M., Wimmer, H., 2015. Accessing orthographic representations from speech: the role of left ventral occipitotemporal cortex in spelling. Hum. Brain Mapp. 36, 1393-1406.

Lukatela, G., Turvey, M.T., 1994. Visual lexical access is initially phonological: i. Evidence from associative priming by words, homophones, and pseudohomophones. J. Exp. Psychol. Gen. 123, 107-128.

Mason, M.F., Norton, M.I., Van Horn, J.D., Wegner, D.M., Grafton, S.T., Macrae, C.N. 2007. Wandering minds: the default network and stimulus-independent thought. Science 315 (80-), 393-395.

McDermott, K.B., Petersen, S.E., Watson, J.M., Ojemann, J.G., 2003. A procedure for identifying regions preferentially activated by attention to semantic and phonological relations using functional magnetic resonance imaging. Neuropsychologia 41, 293-303.

Mechelli, A., Josephs, O., Ralph, L., Matthew, A., McClelland, J.L., Price, C.J., 2007. Dissociating stimulus-driven semantic and phonological effect during reading and naming. Hum. Brain Mapp. 28, 205-217.

Nieto-Castañón, A., Fedorenko, E., 2012. Subject-specific functional localizers increase sensitivity and functional resolution of multi-subject analyses. Neuroimage 63, 1646-1669.

Ortells, J.J., Kiefer, M., Castillo, A., Megias, M., Morillas, A., 2016. The semantic origin of unconscious priming: behavioral and event-related potential evidence during category congruency priming from strongly and weakly related masked words. Cognition 146, 143-157.

Poldrack, R.A., Wagner, A.D., Prull, M.W., Desmond, J.E., Glover, G.H., Gabrieli, J.D.E., 1999. Functional specialization for semantic and phonological processing in the left inferior prefrontal cortex. Neuroimage 10, 15-35.

Price, C.J., 2012. A review and synthesis of the first 20 years of PET and fMRI studies of heard speech, spoken language and reading. Neuroimage 62, 816-847.

Price, C.J., Devlin, J.T., 2011. The Interactive Account of ventral occipitotemporal contributions to reading. Trends Cogn. Sci. 15, 246-253.

Price, C.J., Friston, K.J., 1997. The temporal dynamics of reading: a PET study. Proc. R. Soc. Lond. B Biol. Sci. 264, 1785-1791.

Price, C.J., Mechelli, A., 2005. Reading and reading disturbance. Curr. Opin. Neurobiol. $15,231-238$.

Price, C.J., Moore, C.J., Humphreys, G.W., Wise, R.J.S., 1997. Segregating semantic from phonological processes during reading. J. Cogn. Neurosci. 9, 727-733.

Raichle, M.E., MacLeod, A.M., Snyder, A.Z., Powers, W.J., Gusnard, D.A., Shulman, G.L., 2001. A default mode of brain function. Proc. Natl. Acad. Sci. 98, 676-682.

Riecker, A., Ackermann, H., Wildgruber, D., Dogil, G., Grodd, W., 2000. Opposite hemispheric lateralization effects during speaking and singing at motor cortex, insula and cerebellum. Neuroreport 11, 1997-2000.

Rodd, J.M., 2004. When do leotards get their spots? Semantic activation of lexical neighbors in visual word recognition. Psychon. Bull. Rev. 11, 434-439. http:// 
dx.doi.org/10.3758/BF03196591.

Rueckl, J.G., Paz-Alonso, P.M., Molfese, P.J., Kuo, W.-J., Bick, A., Frost, S.J., Hancock, R., Wu, D.H., Mencl, W.E., Dunabeitia, J.A., Lee, J.-R., Oliver, M., Zevin, J.D., Hoeft, F., Carreiras, M., Tzeng, O.J.L., Pugh, K.R., Frost, R., 2015. Universal brain signature of proficient reading: evidence from four contrasting languages. Proc. Natl. Acad. Sci.. http://dx.doi.org/10.1073/pnas.1509321112.

Rumsey, J.M., Horwitz, B., Donohue, B.C., Nace, K., Maisog, J.M., Andreason, P., 1997. Phonological and orthographic components of word recognition. A PET-rCBF study. Brain 120, 739 .

Sarter, M., Givens, B., Bruno, J.P., 2001. The cognitive neuroscience of sustained attention: where top-down meets bottom-up. Brain Res. Rev. 35, 146-160.

Seidenberg, M.S., McClelland, J.L., 1989. A distributed, developmental model of word recognition and naming. Psychol. Rev. 96, 523-568.

Tanenhaus, M.K., Flanigan, H.P., Seidenberg, M.S., 1980. Orthographic and phonological activation in auditory and visual word recognition. Mem. Cogn. 8, $513-520$.

Thiebaut De Schotten, M., Cohen, L., Amemiya, E., Braga, L.W., Dehaene, S., 2014 Learning to read improves the structure of the arcuate fasciculus. Cereb. Cortex 24, 989-995.

Twomey, T., Kawabata Duncan, K.J., Price, C.J., Devlin, J.T., 2011. Top-down modulation of ventral occipito-temporal responses during visual word recognition. Neuroimage 55, 1242-1251.

Vagharchakian, L., Dehaene-Lambertz, G., Pallier, C., Dehaene, S., 2012. A temporal bottleneck in the language comprehension network. J. Neurosci. 32, 9089-9102.

Van Orden, G.C., 1987. A ROWS is a ROSE: spelling, sound, and reading. Mem. Cogn. 15, 181-198.

Van Orden, G.C., Goldinger, S.D., 1994. Interdependence of form and function in cognitive systems explains perception of printed words. J. Exp. Psychol. Hum. Percept. Perform. 20, 1269-1291.
Vinckier, F., Dehaene, S., Jobert, A., Dubus, J.P., Sigman, M., Cohen, L., 2007. Hierarchical coding of letter strings in the ventral stream: dissecting the inner organization of the visual word-form system. Neuron 55, 143-156.

Wagner, A.D., Koutstaal, W., Maril, A., Schacter, D.L., Buckner, R.L., 2000. Task-specific repetition priming in left inferior prefrontal cortex. Cereb. Cortex 10, 1176-1184.

Wheat, K.L., Cornelissen, P.L., Frost, S.J., Hansen, P.C., 2010. During visual word recognition, phonology is accessed within $100 \mathrm{~ms}$ and may be mediated by a speech production code: evidence from magnetoencephalography. J. Neurosci. 30, 5229-5233.

Wilson, L.B., Tregellas, J.R., Slason, E., Pasko, B.E., Rojas, D.C., 2011. Implicit phonological priming during visual word recognition. Neuroimage 55, 724-731.

Wise, R.J.S., Greene, J., Büchel, C., Scott, S.K., 1999. Brain regions involved in articulation. Lancet 353, 1057-1061.

Woodhead, ZV.J., Barnes, G.R., Penny, W., Moran, R., Teki, S., Price, C.J, Leff, A.P., 2014. Reading front to back: meg evidence for early feedback effects during word recognition. Cereb. Cortex 24, 817-825.

Yang, J., Wang, X., Shu, H., Zevin, J.D., 2012. Task by stimulus interactions in brain responses during Chinese character processing. Neuroimage 60, 979-990.

Yeatman, J.D., Dougherty, R.F., Rykhlevskaia, E., Sherbondy, A.J., Deutsch, G.K. Wandell, B.A., Ben-Shachar, M., 2011. Anatomical properties of the arcuate fasciculus predict phonological and reading skills in children. J. Cogn. Neurosci. 23, 3304-3317.

Zekveld, A.A., Heslenfeld, D.J., Festen, J.M., Schoonhoven, R., 2006. Top-down and bottom-up processes in speech comprehension. Neuroimage 32, 1826-1836.

Ziegler, J.C., Jacobs, A.M., 1995. Phonological information provides early sources of constraint in the processing of letter strings. J. Mem. Lang. 34, 567-593.

Ziegler, J.C., Perry, C., Zorzi, M., 2014. Modelling reading development through phonological decoding and self-teaching: implications for dyslexia. Philos. Trans. R. Soc. B Biol. Sci. 369, 20120397. 